\title{
BALANÇO DE RADIAÇÃO NO PANTANAL SUL MATO-GROSSENSE DURANTE A ESTAÇÃO SECA
}

\author{
FRANCISCO LUIZ LEITÃO DE MESQUITA ${ }^{1}$, EDSON PEREIRA MARQUES FILHO ${ }^{1}$, HUGO ABI \\ KARAM $^{1}$, REGINA CÉLIA DOS SANTOS ALVALÁ \\ ${ }^{1}$ Universidade Federal do Rio de Janeiro (UFRJ), Rio de Janeiro, RJ, Brasil \\ ${ }^{2}$ Instituto Nacional de Pesquisas Espaciais (INPE), São José dos Campos, SP, Brasil \\ fllm@oi.com.br, edson@igeo.ufrj.br,hugo@igeo.ufrj.br, regina@cptec.inpe.br
}

Recebido Outubro de 2011 - Aceito Agosto de 2012

\begin{abstract}
RESUMO
Este trabalho apresenta a análise das componentes do balanço de radiação à superfície sobre o Pantanal Sul Mato-grossense, a partir de medidas experimentais coletadas durante a estação seca, em setembro de 1999. Neste período, as componentes do balanço de radiação mostraram um ciclo diurno bem definido, associado à densidade de fluxo radiativo de onda curta de $850 \mathrm{Wm}^{-2}$ ao meio dia. $\mathrm{O}$ albedo médio apresentou um comportamento quase especular, com valor mínimo de $0,16 \pm 0,02$ para ângulos zenitais pequenos. Os valores de emissividade da superfície pantaneira, corrigida para temperatura radiativa de um corpo cinza, variaram entre 0,94 e 0,96 . A partir das medidas diretas das densidades dos fluxos radiativos de ondas curtas e longas atmosféricas determinou-se os parâmetros ótimos empregados nas formulações propostas por Monteith e Unsworth (2008), Swinbank (1963) e Brutsaert (1975).

Palavras-chave: Balanço de radiação, Experimento Interdisciplinar do Pantanal, albedo, parametrizações radiométricas.
\end{abstract}

\begin{abstract}
BUDGET RADIATION ON PANTANAL WETLAND IN MATO GROSSO DO SUL STATE DURING THE DRY SEASON

This work analyses the surface radiation budget components observed over the Pantanal wetland located in the south part of Mato Grosso do Sul State, on September,1999 during the dry season. For this period, these components showed a well defined diurnal cycle, with the shortwave radiation fluxes showing a maximum of $850 \mathrm{Wm}^{-2}$ at noon. The albedo presents a specular behavior with minimum equal to $0.16 \pm 0.02$ for small zenithal angle. The land surface emissivity, corrected to a gray body radiative temperature, shows values between 0.94 and 0.96 . Direct measurements of shortwave and long-wave density radiative fluxes were used to adjust the optimum parameters on the proposed Monteith and Unsworth (2008), Swinbank (1963) and Brutsaert (1975) formulations.

Keywords: Radiation budget, Interdisciplinary Pantanal Experiment, albedo, radiometric parameterizations.
\end{abstract}

\section{INTRODUÇÃO}

O balanço de energia na superfície está associado à dinâmica dos processos de interação superfície-atmosfera e têm um importante papel no sistema climático terrestre, por meio das trocas de calor, umidade, momento e radiação (Stull, 1988).

No último século as atividades antropogênicas contribuíram significativamente para mudanças nas condições climáticas associadas à perspectiva de um aquecimento global sem precedentes (IPCC, 2007). Estas modificações refletem a atual escassez de recursos naturais e a degradação das condições ambientais, particularmente ocasionadas pelo uso intensivo do solo, desmatamento, queima de combustíveis fósseis e processos de urbanização.

A expansão das fronteiras agrícolas na região CentroOeste e Norte do Brasil exercem uma forte influência sobre os recursos naturais disponíveis a sua volta, entre os quais se destacam as florestas tropicais, matas de cerrado, mananciais 
e lençóis subterrâneos (Nobre, 2008). Neste contexto, o frágil bioma pantaneiro é bastante suscetível às modificações em suas características físicas e biogeoquímicas (Hamilton, 2010).

O Pantanal é a maior planície inundada do planeta, com uma área de aproximadamente $160.000 \mathrm{~km}^{2}$, de biodiversidade única e de alta vulnerabilidade (Sawyer, 2009). A principal atividade econômica desta região é a pecuária de corte, favorecida pelas extensas áreas planas de campos naturais (Junk e Cunha, 2005). Porém, outras atividades como agricultura, indústrias químicas e de mineração, além de iniciativas de construção de hidrelétricas e termoelétricas, contribuem para degradação das condições ambientais de maneira irreversível (Costa et al., 2007). Um dos grandes desafios para a preservação do Pantanal é a viabilização de um desenvolvimento sustentável e socialmente responsável (Moraes et al., 2009).

Em razão de sua importância ambiental e econômica, a comunidade científica tem demonstrado grande preocupação com o ecossistema pantaneiro, gerando assim diversos estudos relacionados a este bioma (Vila da Silva e Abdon, 1998; von Randow e Alvalá, 2006; Marques Filho et al., 2008; Lourival et al., 2009; Ariera et al., 2010; Girard et al., 2010). Vila da Silva e Abdon (1998) utilizaram técnicas de sensoriamento remoto para caracterizar a produtividade agropecuária em diferentes sub-regiões do Pantanal. O comportamento da radiação de onda longa emitida pela atmosfera $(O L A)$ durante a estação seca foi investigado por von Randow e Alvalá (2006). As características da turbulência na Camada Limite Superficial (CLS) sobre a região do Pantanal foram exploradas por Marques Filho et al.(2008). Lourival et al. (2009) analisaram a evolução sistemática de diferentes cenários de conservação e verificaram que apenas o plano Reserva da Biosfera apresenta a melhor relação entre eficiência e oportunidade. Ariera et al. (2010) e Girard et al. (2010), utilizando também técnicas de sensoriamento remoto, realizaram o mapeamento da vegetação e a dinâmica de inundações na região do Pantanal.

O objetivo deste trabalho é caracterizar a evolução temporal do balanço de radiação e do albedo sobre a superfície (BRS) do Pantanal Sul Mato-grossense, e propor parametrizações que representem adequadamente as componentes de radiação de onda longa e curta. Como objetivo secundário foi realizado a caracterização do ciclo diurno às condições termodinâmicas inerentes da CLS do Pantanal Sul Mato-grossense.

\section{MATERIAL E MÉTODOS}

\subsection{Elementos teóricos}

A radiação solar ao penetrar na atmosfera sofre processos de reflexão, espalhamento e absorção, decorrentes da cobertura de nuvens, da concentração de vapor de água e de outros aerossóis atmosféricos (Liou, 2002). Apenas uma fração desta radiação atinge diretamente a superfície terrestre, enquanto que o restante representa as contribuições difusas e térmicas da atmosfera (Oke, 1987).

A radiação de onda curta da atmosfera $(O C A)$ é a principal fonte de energia dos processos físicos naturais que ocorrem na interface superfície-atmosfera. Os comprimentos de onda do espectro eletromagnético da $O C A$ variam de $0,1 \mu \mathrm{m}$ a $4 \mu \mathrm{m}$, e correspondem às bandas do ultravioleta, visível e infravermelho próximo (Brutsaert, 1982). Basicamente, o comportamento da $O C A$ está relacionado a parâmetros astronômicos, a localização geográfica e ao ângulo zenital (Muneer, 2004). Monteith e Unsworth (2008) apresentaram o seguinte conjunto de equações para representar o ciclo diurno da $O C A$ em condições de céu claro sobre superfícies continentais:

$$
\begin{aligned}
& O C A_{\text {dir }}=S_{o} \exp \left[-m\left(\tau_{m}+\tau_{a}\right)\right] \cos z \\
& O C A_{\text {dif }}=0,3 S_{o}\left\{1-\exp \left[-m\left(\tau_{m}+\tau_{a}\right)\right]\right\} \cos z \\
& m=\left(p / p_{o}\right) \sec z \\
& O C A=O C A_{d i r}+O C A_{d i f}
\end{aligned}
$$

sendo, $S_{o}$ a constante solar $\left(1367 \mathrm{Wm}^{2}\right), m$ amassa ótica relativa do ar, $\tau_{m}$ e $\tau_{a}$ os coeficientes de atenuação molecular devido aos gases constituintes da atmosfera e aerossóis, $p$ e $p_{0}$ as respectivas pressões ao nível de medida e de referência, $z$ o ângulo zenital, $O C A_{d i r}$ e $O C A_{d i f}$ as frações direta e difusa de $O C A$. O coeficiente $\tau_{m}$ foi assumido igual 0,3 (Monteith e Unsworth, 2008) e o coeficiente $\tau_{a}$ foi estimado em função dos valores medidos de $O C A$ e $p$, de acordo:

$$
\tau_{a}=1 / m\left[\ln \left(S_{0} / O C A\right)\right]-0,3
$$

A parametrização utilizada na estimativa da $O C A$ sobre a superfície pantaneira foi obtida por meio de uma regressão linear entre as medidas diretas de $O C A$ coletadas em superfície e os resultados gerados pela Equação 4.

Outro parâmetro físico importante do BRS é o albedo superficial $(\alpha)$, definido pela razão:

$$
\alpha=O C R / O C A
$$

sendo $O C R$ a radiação de onda curta refletida. O parâmetro $\alpha$ sofre fortes influências das características físicas da superfície do solo (tipo de cobertura, umidade e topografia), do ângulo zenital solar e das condições de nebulosidade da atmosfera (Oke, 1987; Munner, 2004; Arya, 2005). Segundo Oke (1987) e Wang et al. (2007) $\alpha$ desempenha um papel fundamental nas condições microclimáticas locais, pois influencia diretamente a energia disponível na interface superfície-atmosfera para os processos de aquecimento, evapotranspiração e fotossíntese. De acordo 
com Leitão et al. (2002), $\alpha$ também é um importante parâmetro de entrada nos modelos climáticos e agrometeorológicos de previsão do tempo.

A radiação de onda longa é o resultado dos fluxos radiantes de energia emitidos pelos gases constituintes da atmosfera $(O L A)$ e pelas superfícies sólidas e líquidas da Terra $(O L S)$. O comprimento de onda desta componente, também conhecida como radiação termal, varia entre $4 \mu \mathrm{m}$ e $100 \mu \mathrm{m}$ (Brutsaert, 1982).

Pela Lei de Stefan-Boltzmann todos os corpos a uma dada temperatura emitem radiação em diferentes comprimentos de onda do espectro eletromagnético (Liou, 2002). Portanto, a superfície terrestre pode ser considerada como um corpo negro e a $O L S$ estimada por:

$$
O L S=\varepsilon_{S} \sigma T_{S}^{4}
$$

sendo $\varepsilon_{s}$ e $T_{S}$ a emissividade e a temperatura da superfície, respectivamente.

A $O L S$ responde ao ciclo diurno de aquecimento/ resfriamento da superfície e pode ser obtida a partir da Lei de Stefan-Boltzmann (Equação 7). Em razão da grande variabilidade dos gradientes de temperatura do ar nos primeiros centímetros da CLS, é difícil obter medidas diretas de $T_{S}$ (Arya, 2005). Uma alternativa é considerar $T_{S}$ igual à temperatura cinética verdadeira $T_{k i n}$ e associá-la a temperatura radiante aparente $T_{\text {rad }}$ dada pela expressão (Sabins, 1997):

$$
\left(T_{\text {rad }}=\varepsilon_{s}{ }^{1 / 4} T_{k i n}\right)
$$

De acordo com Jensen (2009), uma superfície de solo siltoso úmido e coberto por gramíneas pode ser caracterizada por um valor de emissividade $\varepsilon_{s}$ em torno de 0,96 , associado à diferença média absoluta entre $T_{\text {kin }}$ e $T_{\text {rad }}$ de aproximadamente $3^{\circ} \mathrm{C}$. Portanto, $T_{\text {rad }}$ foi estimada em função da temperatura do solo $\left(T_{g}\right)$ medida a $1 \mathrm{~cm}$ de profundidade, acrescentando-se (diminuindo-se) $3{ }^{\circ} \mathrm{C}$ durante os períodos diurno (noturno). Esta correção de $T_{S}$ é necessária, pois as medidas radiométricas de $O L S$ a partir de um ponto elevado acima da superfície consideram que o fluxo radiativo provém de um corpo negro, o que não é verdade (Jensen, 2009).

A $O L A$ pode ser obtida a partir de medidas diretas, ou estimada por modelos semi-empíricos e de transferência radiativa (von Randow e Alvalá, 2006; Bárbaro et al., 2010). Os modelos de transferência radiativa, além de complexos, requerem informações da estrutura termodinâmica da atmosfera, das concentrações de vapor de água e de outros gases do efeito estufa (Ellingson et al., 1991). Os modelos semi-empíricos são baseados apenas em medidas meteorológicas coletadas em superfície e, portanto, mais fáceis de serem aplicados. Neste estudo os modelos semi-empíricos propostos por Swinbank (1963) (Equação 9) e Brutsaert (1975)
(Equação 10) foram utilizados para representar a evolução diurna da $O L A$ sobre o Pantanal, devido à simplicidade de suas implementações.

Baseado em dados experimentais, Swinbank (1963) propôs a seguinte equação para estimar a $O L A$ em condições de céu claro:

$$
O L A=c_{1} \sigma T^{6}
$$

sendo $c_{1}=9,62 \times 10^{-6}$ o coeficiente de ajuste, $\sigma=5,67 \times 10^{-8}$ $\mathrm{Wm}^{-2} \mathrm{~K}^{-4}$ a constante de Boltzmann e $T$ a temperatura do ar.

Por outro lado, Brutsaert (1975) obteve estimativas da $O L A$ a partir da integração da equação de Schwarzschild sobre todas as direções e comprimentos de onda:

$$
O L A=c_{2}\left(\frac{e}{T}\right)^{\frac{1}{7}} \sigma T^{4}
$$

sendo $c_{2}=1,24$ o coeficiente de ajuste, e $e$ a pressão parcial do vapor de água.

Neste trabalho, as parametrizações de $O L A$ foram ajustados às condições termodinâmicas do Pantanal de acordo com a metodologia apresentada por von Randow e Alvalá (2006) e dadas por:

$$
\begin{aligned}
& O L A=\left[10^{(0,172 \log (T)-0,610)}\right] \sigma T^{4} \\
& O L A=\left[0,108+1,116\left(\frac{e}{T}\right)^{1 / 7}\right] \sigma T^{4}
\end{aligned}
$$

Para avaliar o desempenho das parametrizações empregadas na estimativa de $O C A$ e $O L A$, foi utilizado o coeficiente de determinação $\left(\mathrm{R}^{2}\right)$, o qual indica quanto da variância da grandeza estimada é explicada pela variância da medida, cujo valor varia entre 0 e 1 .

O saldo de radiação líquida $R_{n}$ para uma superfície horizontalmente homogênea e sem capacidade térmica é dado por (Oke, 1987):

$$
R_{n}=O C A+O C R+O L A+O L S
$$

Durante o período diurno, $R_{n}$ apresenta grande amplitude e é dominado pela componente $O C A$, com máximo em torno das $12 \mathrm{~h}$. Enquanto que no período noturno, $R_{n}$ é aproximadamente constante e dominado pelo termo $O L S$.

A determinação da cobertura de nuvens é fundamental para estimativas adequadas das componentes do BRS. As condições de nebulosidade podem ser determinadas em termos da transmissividade atmosférica $(\tau)$, definida como a razão entre $O C A$ e a radiação solar incidente no topo da atmosfera (Monteith e Unsworth, 2008). Nkemdirim (1972) propôs classificar as condições de nebulosidade de acordo com as seguintes classes de transmissividade da atmosfera: $\tau<0,25$ para condições de céu nublado; $0,25 \leq \tau<0,5$ para céu parcialmente nublado; e $0,5 \leq \tau<1$ para condições de céu claro. 


\subsection{Características e climatologia do Pantanal}

O Pantanal é uma planície inundável localizada na região central do Continente Sul Americano, entre latitudes $16^{\circ}$ e $20^{\circ} \mathrm{S}$ e longitudes $58^{\circ}$ e $50^{\circ} \mathrm{W}$, altitude média de $100 \mathrm{~m}$, com extensão territorial de $160.000 \mathrm{~km}^{2}$. Em território brasileiro, sua área é aproximadamente $138.183 \mathrm{~km}^{2}$, correspondente a $85 \%$ da bacia do rio Paraguai (Vila da Silva e Abdon, 1998; Junk e Cunha, 2005).

A planície pantaneira é cortada por inúmeros rios e vazantes, os quais determinam alagamentos periódicos, cuja intensidade, permanência e extensão apresentam variabilidade inter e intra-anual (Gonçalves et al., 2011). As condições ambientais estão associadas a uma enorme biodiversidade. Em áreas de altitude intermediária, onde o solo é arenoso e ácido, encontra-se a vegetação típica do cerrado, com árvores de porte médio (Magalhães, 1992). Em áreas mais baixas e úmidas predominam gramíneas. Matas ciliares estão presentes às margens dos rios, constituídas por árvores de grande e médio porte. Plantas aquáticas de raízes fixas ou flutuantes ocorrem em rios e lagos (Costa et al., 2007).

As mensais climatológicas da temperatura média, mínima e máxima, e umidade relativa do ar média, foram obtidas por análise estatística das medidas do Instituto Nacional de Meteorologia (INMET), coletadas na estação meteorológica de Corumbá-MS (Figuras 1a e 1b). Como esperado, a temperatura média mensal mostra uma evolução sazonal bem definida, com verões quentes e úmidos, e invernos frios e secos. A distribuição mensal média da umidade relativa do ar apresenta uma pequena variabilidade ao longo do ano. A temperatura média anual é aproximadamente $25^{\circ} \mathrm{C}$, com umidade relativa média igual a $75 \%$. A temperatura máxima no verão é superior a $30^{\circ} \mathrm{C}$, com amplitude térmica da ordem de $10^{\circ} \mathrm{C}$, e umidade relativa acima de $78 \%$. No inverno, a queda de temperatura está relacionada à passagem de frentes frias, resultando em mínimas de $18{ }^{\circ} \mathrm{C}$ na média mensal. Mesmo durante este período, considerado seco, a umidade relativa média é superior a $70 \%$, demonstrando a grande disponibilidade de vapor de água.

A Figura 2 apresenta a evolução mensal das componentes médias do balanço hídrico superficial (BHS), obtidas a partir de dados de análise dos NCEP/NCAR (National Centers for Environmental Prediction/National Center for Atmospheric Research), disponíveis para uma grade global com resolução de $2,5^{\circ}$. A série temporal utilizada corresponde ao ponto de grade mais próximo de Corumbá-MS, entre os anos de 1978 e 2009, com resolução mensal (http://www.esrl.noaa.gov/psd/data/reanalysis/).

As componentes do BHS apresentam um regime sazonal bem definido, com excedente de $588,9 \mathrm{~mm}$ entre outubro e abril, e déficit de $398 \mathrm{~mm}$ nos demais meses. Logo, existe uma grande disponibilidade hídrica nos meses excedentes, desempenhando um papel fundamental como fonte de água para os processos hidrometeorológicos da América do Sul (Rao et al., 1996; Hamilton, 2010).

A precipitação anual total é de $1584 \mathrm{~mm}$, com máximos de $280 \pm 58,5$ e $246 \pm 65,1 \mathrm{~mm} \mathrm{mês}^{-1}$ em janeiro e dezembro, respectivamente. As precipitações mínimas concentram-se em julho e agosto, com valores de $11,8 \pm 10,5$ e 9,6 $\pm 6,6 \mathrm{~mm}$ mês ${ }^{-1}$. Em razão deste comportamento pluviométrico, o ciclo hidrológico apresenta duas estações bem distintas: chuvosa e seca. A estação chuvosa está compreendida entre outubro e abril, totalizando $90 \%$ do acumulado, justamente quando ocorrem as inundações das áreas por transbordamento dos principais rios e afluentes que constituem a Bacia do Rio Paraguai.

$\mathrm{Na}$ estação seca, o saldo do BHS é negativo. Tanto as superfícies alagadas, quanto a água contida no solo, sofrem um intenso processo de evaporação devido ao aquecimento superficial, além da transpiração do bioma. A precipitação média neste período equivale a $10 \%$ do volume total anual.
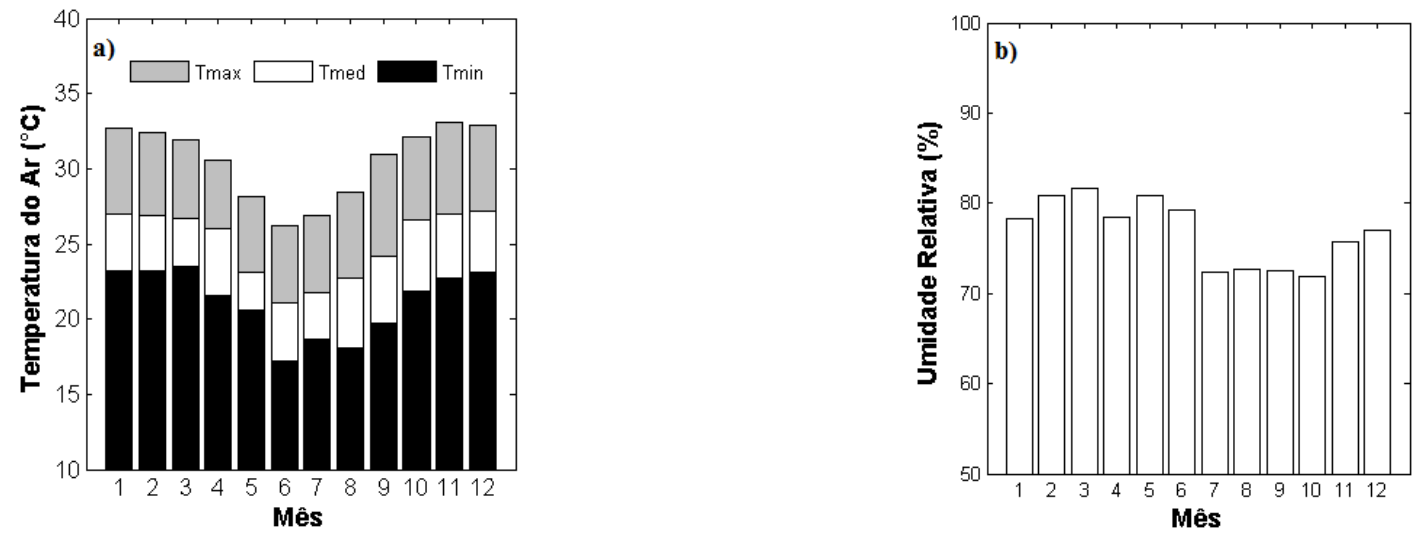

Figura 1 - Médias mensais: (a) das temperaturas máxima (Tmax), média (Tmed) e mínima (Tmin), e (b) umidade relativa para o Pantanal obtidas a partir de medidas do INMET entre o período de 1975 - 1990. 


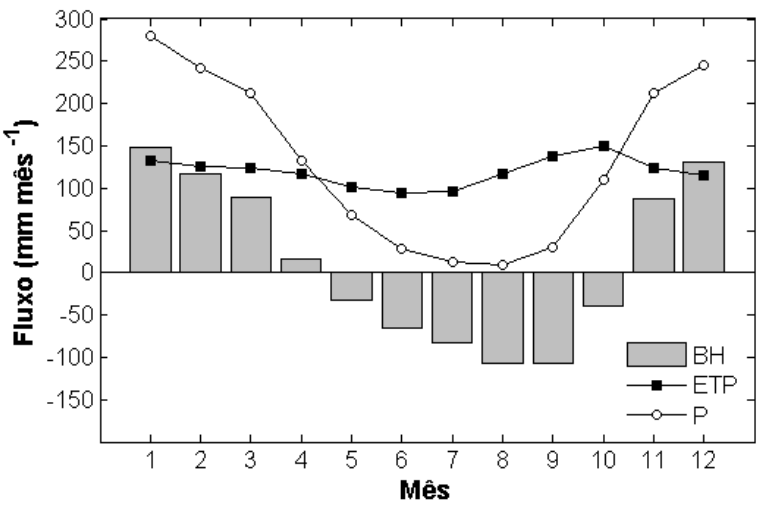

Figura 2 - Balanço hídrico médio mensal (P - precipitação, ETP evapotranspiração de referência e BH - saldo hídrico) para o Pantanal Sul Mato-grossense, derivadas do banco de dados do NCEP/NCAR no ponto de grade mais próximo do município de Corumbá-MS, entre os anos de 1978 e 2009.

A evapotranspiração média corresponde a $68 \%$ do acumulo de água de chuva, com máximo de 149,0 $\mathrm{mm}$ em outubro. As características hídricas obtidas neste estudo concordam com os resultados apresentados por Gonçalves et al. (2011).

\subsection{Sítio experimental e tratamento dos dados}

Para compreender os processos de troca de energia na interface superfície-atmosfera sobre o Pantanal foi realizado um amplo programa experimental denominado Interdisciplinary Pantanal Experiment (IPE). O projeto IPE reuniu esforços do Instituto Nacional de Pesquisas Espaciais (INPE), da Universidade Federal do Mato Grosso do Sul (UFMS) e de outras instituições de ensino e pesquisa. O sítio experimental localizava-se no Pantanal Sul Mato-grossense, próximo à Base de Estudos do Pantanal da UFMS (19³3'48's; 57 00'53”'W), no município de Corumbá-MS (Figura 3). Quatro campanhas intensivas (IPE-0, IPE-1, IPE-2 e IPE-3) foram realizadas entre os anos de 1998 e 2002, e possibilitaram a coleta de um amplo conjunto de medidas micrometeorológicas.

As medidas utilizadas neste estudo (Tabela 1) foram coletadas com razão de amostragem de $0,02 \mathrm{~Hz}$, durante a terceira campanha intensiva (IPE-2) realizada no mês

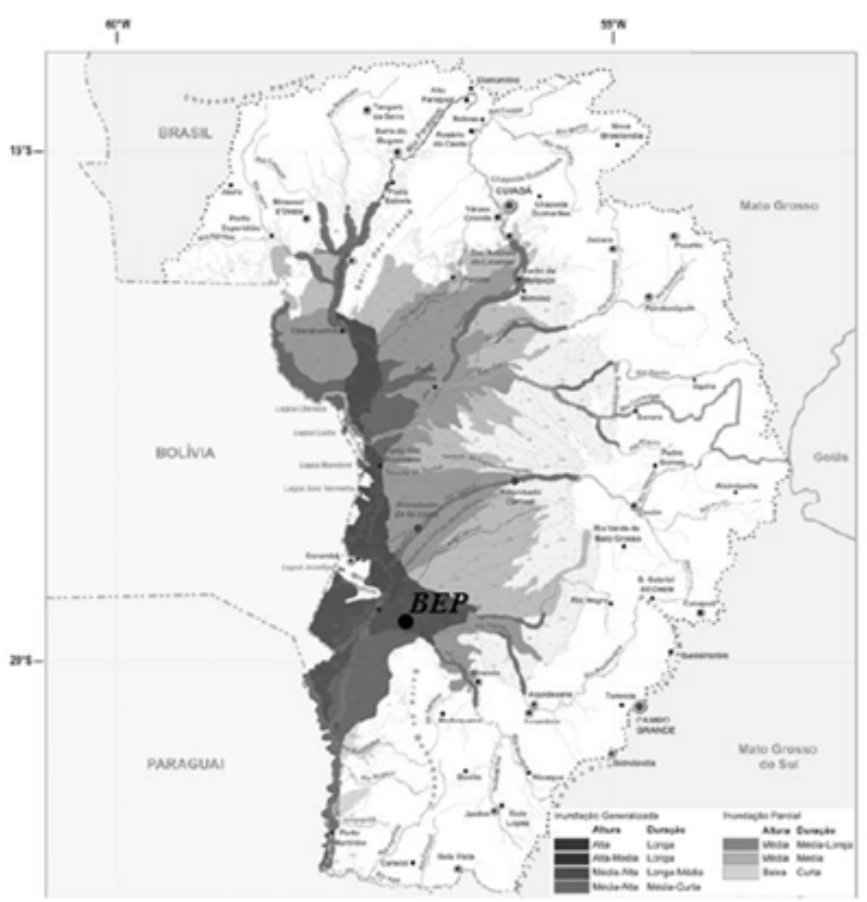

Figura 3 - Distribuição territorial do Pantanal e localização geográfica da Base de Estudos do Pantanal (BEP) da UFMS, Corumbá-MS. FONTE: Adaptada de Agência Nacional de Águas (2011).

de setembro de 1999, entre os dias julianos 261 a 265 . O procedimento adotado para posterior análise dos resultados foi composto de três etapas. Na primeira, as medidas foram submetidas a um controle de qualidade visual e automático para identificar problemas ocorridos durante o registro das séries temporais. Entre estes problemas, "dropout" (Vickers e Mahrt, 1997) decorrentes de falhas eletrônicas do sistema de aquisição de dados. Na segunda, aplicou-se um método estatístico baseado em intervalos de confiança e limites absolutos para remover os valores espúrios (Wilks, 2006). E por último, as formulações de Bolton (1980) e Bohren e Albrecht (1998) foram utilizadas para derivar as variáveis termodinâmicas relevantes. A consistência física destas medidas foi analisada considerando o comportamento das variáveis conservativas em uma Camada Limite Atmosférica (CLA) não saturada.

Após a obtenção do conjunto de dados válidos, recalcularam-se os parâmetros estatísticos a cada 30 minutos,

Tabela 1 - Grandezas meteorológicas medidas no IPE-2 e os respectivos instrumentos

\begin{tabular}{lcccc}
\hline \multicolumn{1}{c}{ Instrumentos } & Fabricante & Variáveis medidas & Unidade & Altura \\
\hline Saldo radiômetro & Kipp\&Zonen & OCA, OCR, OLA,OLS & $\mathrm{Wm}^{-2}$ & $4 \mathrm{~m} \mathrm{e} 22 \mathrm{~m}$ \\
Barômetro & Vaisala & $P$ & $\mathrm{~Pa}$ & $12 \mathrm{~m}$ \\
Psicrômetro & Campbell & $T$ & $\mathrm{~K}$ & $8 \mathrm{~m}$ \\
Razão de Bowen & Licor & $T, q(*)$ & $\mathrm{K}, \mathrm{kg} \mathrm{kg}^{-1}$ & $2 \mathrm{~m} \mathrm{e} 4 \mathrm{~m}$ \\
Termistores & Campbell & $T_{s}$ & $\mathrm{~K}$ & $1 \mathrm{~cm} \mathrm{e} 2 \mathrm{~cm}$ \\
\hline
\end{tabular}

$(*)$ a umidade específica do ar $(q)$ foi utilizada para determinar a pressão parcial de vapor $(e)$. 
período que corresponde a escala integral de tempo na CLS (Wingaard, 1992; Kaimal e Finnigan, 1994). Em particular, as componentes do BRS foram calculadas para a condição média de céu claro de acordo com o critério proposto por Nkemdirim (1972).

Maiores detalhes sobre as características dinâmicas e termodinâmicas da atmosfera local, em diferentes escalas, observadas durante o IPE-2, podem ser obtidas em Domingues et al. (2004).

\section{RESULTADOS}

As evoluções temporais médias para cada 30 minutos das variáveis termodinâmicas próxima da superfície são apresentadas na Figura 4. Apesar da variabilidade das estimativas, os valores médios de $q$ ficaram em torno de $14 \mathrm{~g}$ $\mathrm{kg}^{-1}$. O aumento de $q$ entre $6 \mathrm{~h}$ e $8 \mathrm{~h}$, reflete a contribuição dos processos de evapotranspiração decorrentes do aquecimento da superfície pela $O C A$.

A temperatura potencial virtual $\left(\theta_{v}\right)$ apresentou um ciclo diurno bem definido, em resposta ao aquecimento (resfriamento) radiativo da superfície pela $O C A(O L S)$. O valor médio de $\theta_{v}$ foi igual a $26,7^{\circ} \mathrm{C}$, com amplitude máxima $12,8^{\circ} \mathrm{C}$ próxima às $15 \mathrm{~h}$. Uma característica importante de $\theta_{v}$ está relacionada às suas propriedades conservativas durante os processos adiabáticos não-saturados, além de considerar as influências da umidade na força de empuxo das parcelas de ar, uma vez que o ar úmido é mais leve que o ar seco (Stull, 1988).

Estes resultados demonstram que durante o IPE-2, o Pantanal Sul Mato-grossense apresenta características termodinâmicas similares às encontradas em uma região tropical úmida, com grande disponibilidade hídrica e razão de Bowen média de 0,25 (Gonçalves et al., 2011; Marques Filho et al., 2011).

O ciclo diurno do BRS durante o IPE-2 é apresentado na Figura 5. O sinal dos fluxos radiativos segue a notação matemática, onde o negativo indica o sentido atmosferasuperfície e vice-versa. Nota-se que $O C A$ e $R_{n}$ atingem valores máximos respectivos de $845 \mathrm{Wm}^{-2}$ e $543 \mathrm{Wm}^{-2}$ as $12 \mathrm{~h}$. A $O L S$ apresentou valor máximo de $501,5 \mathrm{Wm}^{-2}$ às $15 \mathrm{~h}$, conjuntamente com o máximo de $T_{S}$ neste mesmo horário, conforme a lei de Stefan-Boltzmann. O máximo registrado para a $O L A$ ocorreu as $14 \mathrm{~h}$, cujo valor foi de $442 \mathrm{Wm}^{-2}$.

O conhecimento do comportamento de $O C A$ é necessário em diversas áreas da atividade humana. A seleção e o cultivo de culturas agrícolas, a otimização do conforto ambiental e a eficiência energética de habitações são exemplos relevantes da aplicação desse conhecimento (Martins e Pereira, 2011). Para exemplificar a importância de $O C A$ no BRS, calculou-se as médias de $O C A$ e $R_{n}$ para o intervalo compreendido entre as $6 \mathrm{~h}$ e $18 \mathrm{~h}$, e posteriormente determinou-se a fração $R_{n} / O C A$, cujo valor obtido foi de 0,68 , próximo aos encontrados por Shuttleworth (1988), Giambelluca et al. (1997) e Galvão e Fisch (2000) para a região da floresta Amazônica.

A evolução temporal do albedo $(\alpha)$ é inversamente proporcional a $O C A$ (Figura 6), ou seja, menores valores de $\alpha$ implicam em maior quantidade de energia radiante absorvida pela superfície. Na área do sítio experimental, o valor médio de $\alpha$ no período de maior incidência solar, entre $10 \mathrm{~h}$ e $15 \mathrm{~h}$, foi aproximadamente igual a 0,16. Para ângulos zenitais maiores, no nascer e pôr do Sol, $\alpha$ aumenta de forma significativa. Este comportamento quase especular do albedo, representado pelo ajuste polinomial de $2^{\mathrm{a}}$ ordem (Figura 6), pode ser associado às condições de homogeneidade horizontal da superfície (Kondratyev, 1969; Iqbal, 1983; Wallace e Robbs, 2006; Monteith e Unsworth, 2008). O valor médio de $\alpha$ ao longo do período diurno foi igual a $0,20 \pm 0,05$. Comparativamente a outros biomas brasileiros, o valor de $\alpha$ para o Pantanal apresenta ordem de magnitude similar ao 0,20 para a região da Floresta Amazônica (Souza et al., 2010) e 0,25 para região de Caatinga (Silva et al., 2005).

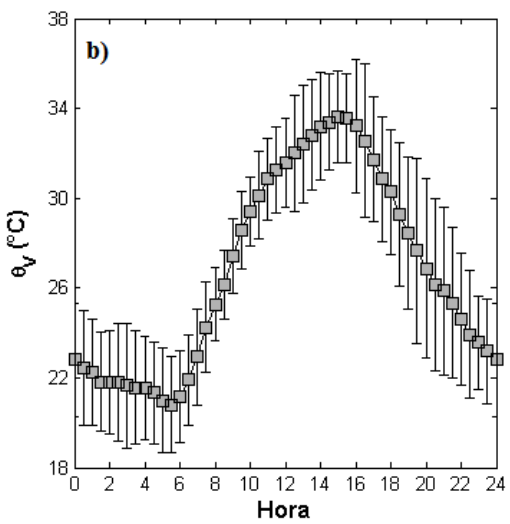

Figura 4 - Evolução temporal diária das médias de 30 minutos com os respectivos desvios-padrão da (a) umidade específica ( $\mathrm{g} \mathrm{kg}^{-1}$ ) e (b) temperatura potencial virtual $\left({ }^{\circ} \mathrm{C}\right)$. 


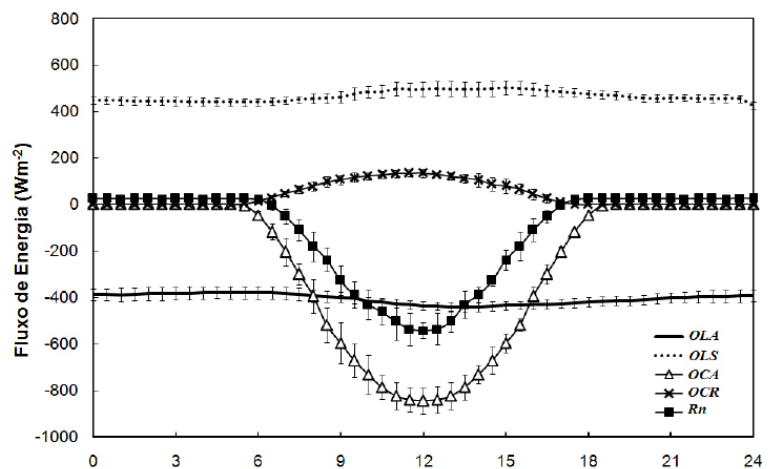

Figura 5 - Evolução temporal diária das médias de 30 minutos das componentes do balanço de radiação à superfície durante o IPE2. Os símbolos representam as médias e as barras verticais seus respectivos desvios padrão. O sinal dos fluxos radiativos segue a notação matemática, sendo os negativos (positivos) indicam o sentido atmosfera-superfície (superfície-atmosfera).

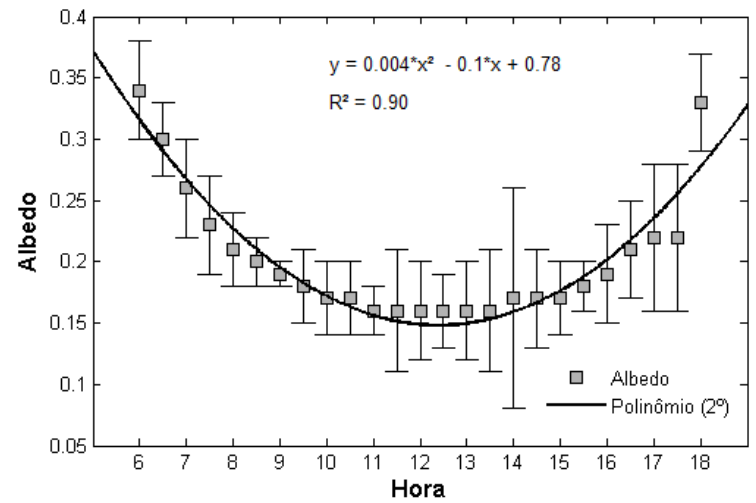

Figura 6 - Evolução temporal diária das médias de 30 minutos do albedo da superfície. Os quadrados e a linha contínua representam respectivamente as médias e a linha de tendência polinomial. As barras verticais correspondem aos desvios-padrão.

O comportamento da $O C A$ sobre o Pantanal foi modelado de acordo com as Equações 1-4, o que permitiu comparar os resultados simulados e observados (Figura 7). O coeficiente de determinação $\mathrm{R}^{2}$ foi igual a 0,971 , indicando que $97,10 \%$ da variância de $O C A$ estimada é explicada pela variância de $O C A$ medida. Conseqüentemente, nota-se uma forte correlação entre as estimativas e as medidas de $O C A$ ao longo de todo intervalo $(\mathrm{R}=0,985)$. $\mathrm{O}$ espalhamento nas estimativas de $O C A$, para valores inferiores a $200 \mathrm{Wm}^{-2}$, pode estar associado à fração difusa da radiação solar devido ao aumento do caminho ótico nos períodos de transição (Iqbal, 1983; Liou, 2002).

Comparativamente à $O C A$, a evolução temporal das componentes $O L S$ e $O L A$ são aproximadamente constantes durante todo o dia (Figura 5). O conhecimento do balanço de onda longa $(O L=O L A+O L S)$ é importante para caracterização

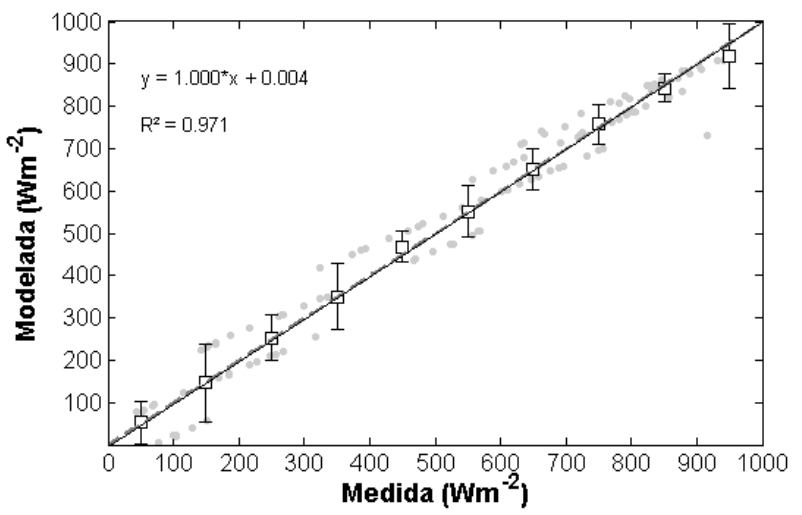

Figura 7 - Radiação de onda curta incidente $(O C A)$, medida e estimada pelo modelo de Monteith e Unsworth ajustado. Os círculos em cinza representam os valores médios para cada 30 minutos, os quadrados em branco são as médias dos modelos para intervalos de $100 \mathrm{Wm}^{-2} \mathrm{de}$ $O C A$ e as barras verticais seus respectivos desvios-padrão.

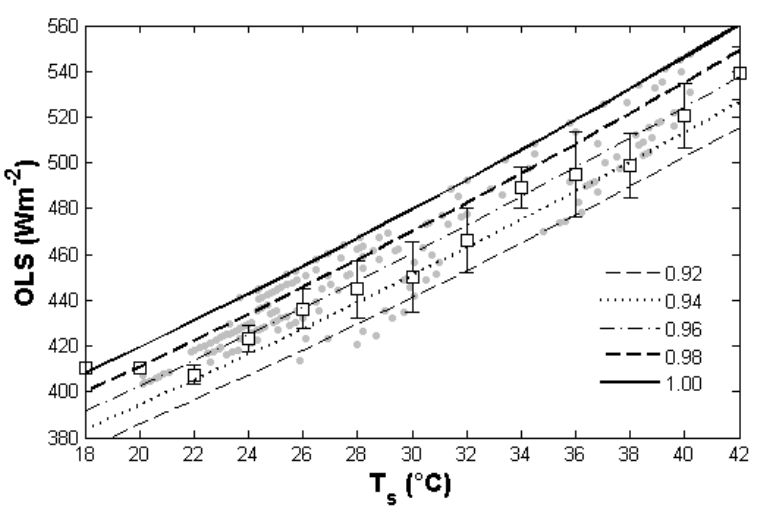

Figura 8 - Variação da $O L S$ em função de $T_{S}$, considerada igual à temperatura de brilho. Os círculos em cinza representam as médias de 30 minutos durante todo o período, os quadrados em branco são as médias de $O L S$ para intervalos de $2{ }^{\circ} \mathrm{C}$ de $T_{S}$, com seus respectivos desvios-padrão (barras verticais). As linhas representam diferentes valores de emissividade da superfície (linha contínua corresponde a emissividade do corpo negro).

das trocas de energia na interface superfície-atmosfera durante o período noturno. Nessa região a perda radiativa decorrente de $O L S$ é superior a $O L A$ em noites de céu sem cobertura de nuvens. Nas estações mais frias do ano, esta condição favorece a ocorrência de nevoeiros e geadas, com grande impacto na produção agrícola e nos sistemas de transportes aéreo e rodoviário (Jimenez et al., 1987). Porém, em noites de céu nublado a $O L A$ pode ser superior a $O L S$, devido aos processos de reflexão e emissão ocasionados pela presença de nuvens (Oke, 1987).

Durante o IPE-2 os valores médios diários da emissividade e da temperatura da superfície foram superiores aos encontrados para a CLS. Logo, pela Lei de Stefann-Boltzman, o valor médio diário de $O L$ é positivo e igual a $60 \mathrm{Wm}^{-2}$. 

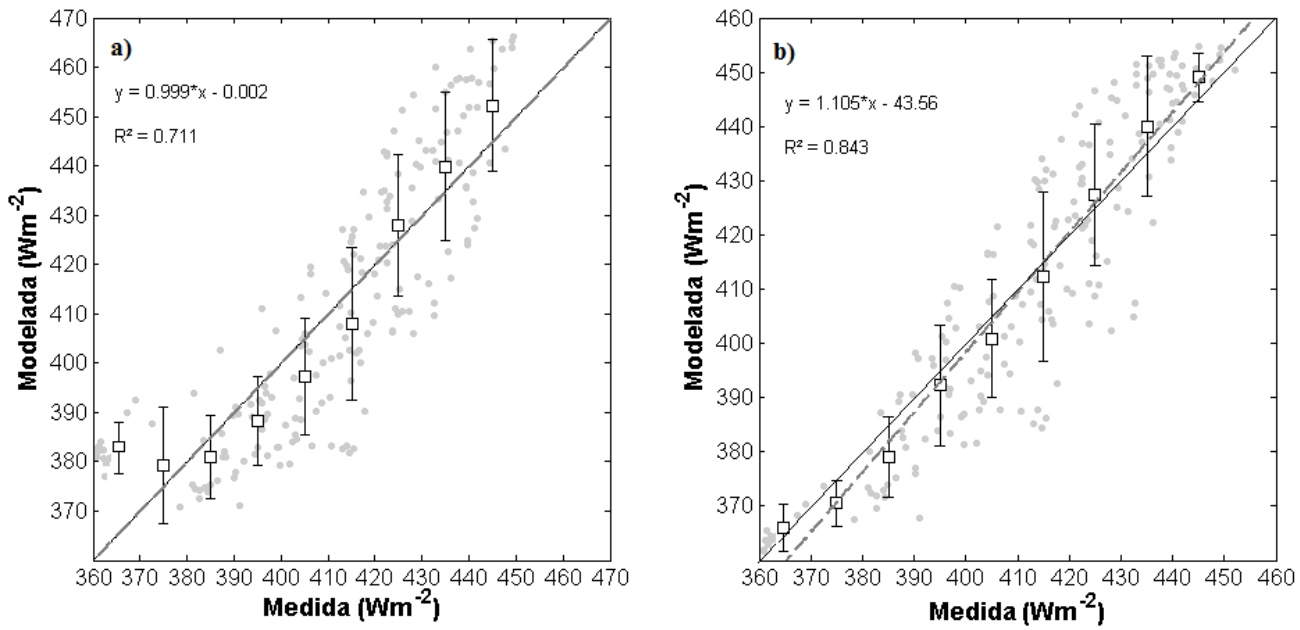

Figura 9 - Radiação de onda longa emitida pela atmosfera $(O L A)$, medida e modelada: (a) Swinbank e (b) Brutsaert. Os círculos em cinza representam os valores médios para cada 30 minutos, os quadrados em branco são as médias dos modelos para intervalos de $10 \mathrm{Wm}^{-2}$ de $O L A$ e as barras verticais seus respectivos desvios.

Apesar da variação intrínseca, a emissividade média da superfície $\varepsilon_{s}$ está compreendida no intervalo $0,94 \leq \varepsilon_{s} \leq 0,96$ (Figura 8). Estes valores de $\varepsilon_{s}$ concordam com as estimativas obtidas por Prigent et al. (2006) por sensoriamento remoto para a mesma localidade, e são superiores aos obtidos por Bastos et al. (2000) para médias de pixel da região do Pantanal Sul

Mato-grossense.

Os modelos semi-empíricos propostos por Swinbank (1963) e Brutsaert (1975), ajustados às condições termodinâmicas da CLS do Pantanal, reproduziram adequadamente o comportamento da $O L A$ medida em superfície (Figuras 9a e $9 b)$. Os coeficientes de determinação $\left(\mathrm{R}^{2}\right)$ foram iguais a 0,711 para Swinbank e 0,843 para Brutsaert, sendo que os coeficientes ajustados para Swinbank (Equação 11) são similares aos encontrados por von Randow e Alvalá (2006), para a mesma área de estudo. As discordâncias nas estimativas de $O L A$, abaixo de $380 \mathrm{Wm}^{-2}$ e acima de $440 \mathrm{Wm}^{-2}$, podem estar relacionadas à concentração de vapor de água na baixa atmosfera (Niemelä et al., 2001). Isto justifica o melhor desempenho do modelo ajustado de Brutsaert, que considera as medidas da pressão parcial do vapor nas estimativas da $O L A$. De acordo com Culf e Gash (1993) e Prata (1996), em regiões quentes e úmidas este modelo apresenta melhor desempenho, como confirmado para o Pantanal.

\section{CONCLUSÕES}

Neste trabalho foi realizado um estudo sobre o BRS na região do Pantanal Sul Mato-grossense a partir de medidas diretas coletadas durante os IPE-2, referente aos dias julianos 261 a 265, na estação seca de setembro de 1999.

As componentes do BRS apresentaram um ciclo diurno bem definido com máximos às $12 \mathrm{~h}\left(O C A, O C R \mathrm{e} R_{n}\right), 14 \mathrm{~h}(O L A)$ e $15 \mathrm{~h}(O L S)$. A $O C A$ mostrou-se ser a componente que mais contribui na geração de $R_{n}(68 \%$ de $O C A)$ durante o período diurno. A evolução temporal de $R_{n}$ responde aos balanços de radiação de ondas curtas durante o período diurno (aquecimento da superfície) e de radiação de onda longa no período noturno (resfriamento da superfície).

A emissividade da superfície, corrigida para temperatura de um corpo cinza, variou entre 0,94 e 0,96 , e foi superior a da CLS. Em razão disto, o balanço de onda longa apresentou valor positivo, com média diária de $60 \mathrm{Wm}^{-2}$. O albedo da superfície possui um comportamento quase especular, com valor mínimo de $0,16 \pm 0,02$ para ângulos zenitais baixos e valor médio diário de $0,20 \pm 0,05$, similar em magnitude para as regiões de Caatinga e Floresta Amazônica.

O modelo discutido por Monteith e Unsworth ajustado as condições do Pantanal reproduziu as observações da $O C A$, com coeficiente de determinação $\mathrm{R}^{2}$ superior a 0,97 . A parametrização de $O L A$ proposta por Brutsaert e ajustada para as condições do Pantanal, apresentou melhor desempenho comparativamente a de Swinbank, e pode ser utilizada para simular o comportamento médio horário desta componente.

\section{AGRADECIMENTOS}

Ao CNPq (Proc. $n^{\circ}$ 476599/2009-1), a FAPERJ (Proc. $\mathrm{n}^{\mathrm{o}}$ E-26/102.394/2010) e a FAPESP pelo suporte financeiro. Aos pesquisadores Leonardo D. A. Sá (INPE), Antônio O. Manzi (INPA) e Amaury de Souza (UFMS), que trabalharam ativamente na coordenação do projeto IPE. Ao INPE, a UFMS, ao INMET e ao NCEP/NCAR por cederem gentilmente os dados meteorológicos utilizados neste estudo. 


\section{REFERÊNCIAS BLIOGRÁFICAS}

AGÊNCIA NACIONAL DE ÁGUAS (ANA). Disponível em: $<$ www.ana.gov.br/Paginas/portais/bacias $>$. Acessado em fevereiro de 2011.

ARIEIRA, J. et al. Integrating field sampling, spatial statistics and remote sensing to map wetland vegetation in the Pantanal, Brazil. Biogeosciences Discussion, v. 7, p. 6889-6934. 2010. doi:10.5194/bgd-7-6889, 2010.

ARYA, S. P. Micrometeorology and atmospheric boundary layer. Pure and Apllied Geophysics, v.162, p. 1721-1745, 2005.

BÁRBARO E. et al.. Observational characterization of the downward atmospheric longwave radiation at the surface in the city of São Paulo. Journal of Applied Meteorology and Climatology,v. 49, n. 12, 2574-259. 2010.

BASTOS, E. J. B.; SOUZA, R. A. F.; AlvAlA, R. C. S. Emissividade da superfície sobre Brasil a partir de observações do SSM/I em $19 \mathrm{GHz}$ e $85 \mathrm{GHz}$. Revista Brasileira de Geofísica, São Paulo, v. 18, n. 2, p. 147-160, 2000.

BOLTON, D. The computation of equivalent potential temperature. Monthly Weather Review, v. 108, p.10461053, 1980.

BOHREN, C. F.; ALBRECHT, B. A. Atmospheric thermodynamics. Oxford University Press, New York, p.402, 1998.

BRUTSAERT, W. On a derivable formula for long-wave radiation from clear skies.Water Resources Research, v. 11, n. 5, p. 742-744, 1975.

BRUTSAERT, W. Evaporation into the atmosphere: theory, history and applications. Dordrecth. D. Reiedel, p.299, 1982.

COSTA, M.; TELMER, K.; EVANS, T. Report: seasonal dynamics of the Pantanal. Department of Geography, University of Victoria, Canada, 2007. Disponível em < http:// geography.uvic.ca/people/faculty/costa.php>.

CULF, A. D., GASH, J. H. C. Longwave radiation from clear skies in Niger: a comparison of observations with simple formulas. Journal of Applied Meteorology v. 32, p. 539-547, 1993.

DOMINGUES, M. O. et al. Análise das condições atmosféricas durante a $2^{\mathrm{a}}$ campanha do experimento interdisciplinar do Pantanal Sul Mato-grossense. Revista Brasileira de Meteorologia, v. 19, n. 1, p.73-88, 2004.

ELLINGSON, R. G.; J. ELLIS AND S. FELS. Intercomparison of radiation codes used to climate models: Long wave results. Journal of Geophysical Research: Atmospheres, v. 96, p. 8929-8963, 1991.

GALVÃO, J. A. C.; FISCH, G. Balanço de energia em áreas de floresta e de pastagem na Amazônia (Jí-Paraná, RO). Revista Brasileira de Meteorologia, v. 15, n. 2, p. 25-37,2000.

GIAMBELLUCA, T. W. et al. Observations of albedo and radiation balance over postforest land surfaces in the eastern
Amazon basin.Journal of Climate. v. 10, p. 919-928, 1997. GIRARD, P. et al. Small-scale spatial variation of inundation dynamics in a floodplain of the Pantanal (Brazil).Springer Science + Business Media b.v.p. 11.doi: 10.1007/s10750009-0046-9, 2010.

GONÇALVES, H. C.; MERCANTE, M. A.; SANTOS, E. T. Hydrological cycle. Brazilian Journal of Biology, v. 71, p. 241-253, 2011.

HAMILTON, S. K. Biogeochemical implications of climate change for tropical rivers and floodplains. Springer Science + Business Media b.v. p 17, 2010. doi: 10.1007/s10750009 0086-1.

INTERGOVERNMENTAL PANEL ON CLIMATE CHANGE. Contribution of Working Group I. Cambridge University Press, Cambridge, UK and New York, NY, USA, p. 996, 2007.

IQBAL, M. An introduction to solar radiation, Toronto: Academic Press. p. 390, 1983.

JENSEN, J. R. Sensoriamento remoto do ambiente: Uma perspectiva em recursos terrestres. Editora Parêntese, São José dos Campos, SP, p. 598, 2009.

JIMENEZ, J. I. et al. On the estimation of longwave radiation flux from clear skies. Theoretical and Applied Climatology, v. 38, p. 3742, 1987.

JUNK, W. J.; CUNHA, N. C. Pantanal: a large South American wetland at a crossroads. Ecological Engineering, v. 24, p. 391-401, 2005.

KAIMAL, J. C.; FINNIGAN, J. J. Atmospheric boundary layer flows: Their structure and measurement. New York: Oxford-University, p. 289, 1994.

KONDRATYIEV, K. Y. Radiation in the Atmosphere, Academic Press, New York. p. 912, 1969.

LEITÃO, M. M. V. B. R.; SANTOS, J. M.; OLIVEIRA, G. M. Estimativas do albedo em três ecossistemas da floresta amazônica. Revista Brasileira de Engenharia Agrícola e Ambiental, v. 6, n. 2, p. 256-261, 2002.

LIOU, K. N. An introduction to atmospheric radiation. International Geophysics Series, vo. 84, New York, p.583, 2002.

LOURIVAL, R. et al. A systematic evaluation of the conservation plans for the Pantanal wetland in Brazil. Wetlands, v. 29, n. 4, 2009.

MAGALHÃES, N. W. Conheça o Pantanal. São Paulo. Editora Terragraph, p.390, 1992.

MARQUES FILHO, E. P. et al. Atmospheric surface layer characteristics of turbulence above the Pantanal wetland regarding the similarity theory. Agricultural and forest meteorology, v. 148, p. 883-892, 2008.

MARQUES FILHO, E. P. et al. Razão de Bowen sobre o Pantanal. Anais do VII Workshop Brasileiro de Micrometeorologia, Santa Maria-RS. 2011. 
MARTINS, F. R.; PEREIRA, E. B. Estudo comparativo da confiabilidade de estimativas de irradiação solar para o sudeste brasileiro obtidas a partir de dados de satélite e por interpolação/extrapolação de dados de superfície. Revista Brasileira de Geofísica, v.29, n.2, p. 265-276, 2011.

MONTHEIT, J. L.; UNSWORTH, M. H. Principles of environmental physics $3^{\text {rd }}$ edition, Oxford: Academic Press, p.420, 2008.

MORAES, S. A.; SAMPAIO, Y.; SEIDL, A. Quanto vale o Pantanal? A valoração ambiental aplicada ao bioma pantanal - dados eletrônicos - Corumbá: Embrapa Pantanal, p.34 (Documentos / Embrapa Pantanal, ISSN 1981-7223; v. 105, 2009.

MUNEER, T. Solar radiation and daylight models $2^{\text {nd }}$ edition, Amsterdam. Elsevier, p. 390, 2004.

NIEMELÄ, S.; RÄISÄNEN, P.; SAVIJÄVI H. Comparison of surface radiative flux parameterizations Part I: longwave radiation. Atmospheric Research, v. 58, p. 1-18, 2001.

NKEMDIRIM, L. C. A note on the albedo of surfaces. Journal of Applied Meteorology, v. 11, n. 5, p. 867-874, 1972.

NOBRE, C. Mudanças Climáticas e o Brasil - contextualização. Parcerias Estratégicas, Brasília, DF n.27, 2008.

OKE, T. R. Boundary Layer Climates. London: Routledge. p.435, 1987.

PRATA, A. J. A new long-wave formula for estimating downward clear-sky radiation at the surface. Quarterly Journal of the Royal Meteorological Society, v. 122, 1127-1158, 1996.

PRIGENT, C.; AIRES, F.; ROSSOW, W. B. Land surface microwave emissivities over the globe for a decade. Bulletin American Meteorological Society, p. 1573-1584, 2006.

RAO, V. B.; CAVALCANTI, I. F. A. HADA, K. Annual variation of rainfall over Brazil and water vapor characteristics over South. American Journal of Geophysical Research, v. 101, n. d21,p.26539-26551, 1996.

SABINS, F. F. Remote sensing principles and interpretation. Freeman, New York, p. 494, 1997.

SHUTTLEWORTH, W. J. Evaporation from Amazonian rainforest, Proceedings of the Royal Society, London, Ser. B, 233, 321-346, 1988.
SILVA, B.; LOPES, M. L.; AZEVEDO, P. V. Determinação do albedo de áreas irrigadas com base em imagens LANDSAT 5 - TM. Revista Brasileira de Agrometeorologia. v. 13, n.2, p. 201-211, 2005.

SOUZA, P. J. O. P. et al.. Albedo da cultura da soja em área de avanço da fronteira agrícola na Amazônia. Revista Brasileira de Engenharia Agrícola e Ambiental v. 14, n. 1, p. 65-73, 2010.

STULL, R. B. An introduction to boundary layer meteorology. Kluwer, Boston. p. 662, 1988.

SWAYER, D. Fluxos de carbono na Amazônia e no cerrado: um olhar socioecossistêmico. Sociedade e Estado, Brasília, v. 24, n. 1, p. 149-171, 2009.

SWINBANK, W. C. Long-wave radiation from clear skies. Quarterly Journal of the Royal Meteorological Society, v. 89 , n. 381 , p. 339-348, 1963.

VILADA SILVA, J. S.; ABDON, M. M. Delimitação do Pantanal brasileiro e suas sub-regiões. Pesquisa Agropecuária Brasileira. v. 33. n. especial, p. 1703-1711, 1998.

VICKERS, D.; MAHRT. L. Quality control an flux sampling problems for tower and aircraft data. Journal of Atmospheric and Oceanic Technology, v. 14, p. 512-526, 1997.

VON RANDOW, R. C. S.; ALVALÁ, R. C. S. Estimativa da Radiação de Onda Longa Atmosférica no Pantanal Sul Mato-Grossense Durante os Períodos Secos de 1999 e 2000. Revista Brasileira de Meteorologia, v. 21, p. 398-412, 2006.

WALLACE, J. M.; HOBBS, P. V. Atmospheric science: an introductory survey, $2^{\text {nd }}$ ed., International Geophysics Series. Academic Press, Elsevier Inc., p. 483, 2006.

WANG, S.; TRISHCHENKO, A. P.; SUN, X. M. Simulation of canopy radiation transfer and surface albedo in the EALCO model. Climate Dynamics, v. 29, p. 615-632, 2007.

WILKS, D. S. Statistical Methods in the Atmospheric Sciences. International Geophysics Series $2^{\text {nd }}$, p.648, 2006.

WINGAARD, J. C. Atmospheric turbulence. Annual Review of Fluid Mechanics, v. 24, p. 205-233, 1992. 